\title{
Perspectivas recientes en la Evaluación Neuropsicológica y Comportamental del \\ Trastorno por Déficit de Atención con/sin Hipeactividad
}

\section{Vicente Félix Mateo}

Investigador del Hospital Clínico de Valencia. Psicopedagogo Secundaria.

España

psicofelix@hotmail.com 


\section{Resumen}

En este artículo se muestran las nuevas tendencias en la evaluación del trastorno por déficit de atención con hiperactividad (TDAH). Partiendo de la valoración más tradicional basada en estimaciones comportamentales realizadas en contextos naturales por padres y profesores, pasando por la evaluación neuropsicológica de las funciones ejecutivas y planteando los retos de la Resonancia Magnética Funcional (RMf).

Palabras Clave: Evaluación TDAH, funciones ejecutivas, evaluación neuropsicológica, RMf. 


\section{Introducción}

El TDAH es un trastorno esencialmente crónico, caracterizado por un patrón persistente de inatención, exceso de actividad e impulsividad como características definitorias. Los estudios epidemiológicos muestran que tiene una incidencia del 3-6\% en la población normal (DSM-IV-TR; APA, 2002). Desde que Still (1902) realizase la primera aproximación al trastorno, hablando de un déficit en el control moral, han sido distintas las aproximaciones y conceptualizaciones que hasta la fecha ha tenido el trastorno, como hiperkinesia, disfunción cerebral mínima (Clements, 1966), o más tarde Virginia Douglas (1972) argumentó que la deficiencia básica de los niños hiperactivos no era el excesivo grado de actividad, sino su incapacidad para mantener la atención y su impulsividad. Por lo que respecta a la valoración del mismo, todavía nos queda un largo camino por recorrer en identificar pruebas diagnósticas fiables especialmente por las dificultades que surgen a la hora de diseñar tareas de laboratorio que intenten evaluar los comportamientos de inatención e impulsividad asociados al TDAH. No obstante, disponemos actualmente de un conjunto de conocimientos que nos permiten una aproximación cada vez más ajustada a la identificación de este trastorno.

Las consecuencias personales y sociales de la impulsividad son evidentes dado el alto índice de niños que cada día son diagnosticados bajo el Trastorno por Déficit de Atención con Hiperactividad (TDAH), una manifestación extrema de las dificultades relacionadas con la autorregulación. No obstante, todavía desconocemos muchos aspectos, tanto a nivel teórico como empírico, sobre cómo identificar estos procesos, hecho que nos ha llevado a elaborar este trabajo, con la esperanza de poder arrojar instrumentos de evaluación más precisos, que nos permitan replicar en una situación artificial aspectos relacionados con la impulsividad.

\section{Funciones ejecutivas y TDAH}

El concepto "función" o "funciones ejecutivas" define la actividad de un conjunto de procesos cognitivos vinculada históricamente al funcionamiento de los lóbulos frontales del cerebro. Las observaciones clásicas de pacientes con lesiones cerebrales focales en estas estructuras han revelado el importante papel que ellas juegan en la ejecución de actividades cognitivas de orden superior como la elaboración de programas complejos de conducta, la formulación de metas o la verificación de la acción en curso (Luria, 1973; Stuss 2002). Entre 
las funciones específicas asociadas al concepto podemos resaltar: inhibición, set shifting, memoria de trabajo, planificación y fluencia, basadas en una clasificación empírica (Pennington y Ozonoff, 1996).

Las definiciones de función ejecutiva recogidas en la literatura científica son, por tanto, amplias y difíciles de operacionalizar. Ozonoff y sus colaboradores parten de la siguiente definición de función ejecutiva: "...es el constructo cognitivo usado para describir conductas dirigidas hacia una meta, orientadas hacia el futuro, que se consideran mediadas por los lóbulos frontales. Incluyen la planificación, inhibición de respuestas prepotentes, flexibilidad, búsqueda organizada y memoria de trabajo. Todas las conductas de función ejecutiva comparten la necesidad de desligarse del entorno inmediato o contexto externo para guiar la acción a través de modelos mentales o representaciones internas" (Ozonoff, Strayer, McMahon y Filloux, 1994, p.1015).

Así pues las funciones ejecutivas harían referencia a un conjunto de estrategias que incluirían: (a) Intención de inhibir una respuesta o demorarla para un momento posterior más adecuado; (b) un plan estratégico de secuencias de acción; y (c) una representación mental de la tarea que incluya la información de los estímulos relevantes codificada en la memoria y la meta futura deseada. Estas operaciones se basan en la idea de que sirven para controlar y regular el procesamiento de la información a lo largo del cerebro (Gazzaniga, Ivry y Mangun, 2001). Precisamente, las investigaciones sobre diversas patologías que han aparecido en los últimos años (e.g., en TDAH, autismo, esquizofrenia, trastorno obsesivo compulsivo o Síndrome de Tourette) se han dirigido en particular a evaluar aquellas capacidades que supuestamente integran el mencionado constructo. Entre ellas cabe destacar las siguientes:

- Planificación. Para conseguir la meta propuesta el sujeto debe elaborar y poner en marcha un plan estratégicamente organizado de secuencias de acción. Es necesario puntualizar que la programación no se limita meramente a ordenar conductas motoras, ya que también planificamos nuestros pensamientos con el fin de desarrollar un argumento, aunque no movamos un solo músculo, o recurrimos a ella en procesos de recuperación de la información almacenada en la memoria declarativa (tanto semántica como episódica o perceptiva). 


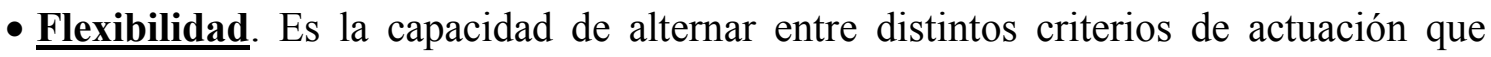
pueden ser necesarios para responder a las demandas cambiantes de una tarea o situación.

- Memoria de trabajo. También llamada memoria operativa. Permite mantener activada una cantidad limitada de información necesaria para guiar la conducta "online", es decir , durante el transcurso de la acción. El sujeto necesita disponer de una representación mental tanto del objetivo como de la información estimular relevante (Ej: el orden en que se han planificado las acciones) no sólo acerca del estado actual sino también en relación a la situación futura. Así, esta capacidad tiene elementos comunes con la memoria prospectiva que implica el recuerdo de la intención de hacer algo. La memoria de trabajo requiere la selección y amplificación de las acciones que son útiles para llevar a cabo la tarea, así como la capacidad de ignorar distratores potenciales (Gazzaniga et al. 2001).

- Monitorización. Es el proceso que discurre paralelo a la realización de una actividad. Consiste en la supervisión necesaria para la ejecución adecuada y eficaz de los procedimientos en curso. La monitorización permite al sujeto darse cuenta de las posibles desviaciones de su conducta sobre la meta deseada. De este modo puede corregirse un posible error antes de ver el resultado final.

- Inhibición. Se refiere a la interrupción de una determinada respuesta que generalmente ha sido automatizada. Por ejemplo, si de repente cambiara el código que rige las señales de los semáforos y tuviéramos que parar ante la luz verde deberíamos inhibir la respuesta dominante o prepotente de continuar la marcha sustituyéndola por otra diferente (en este caso detenernos). La estrategia aprendida, que anteriormente era válida para resolver la tarea, deberá mantenerse en suspenso ante una nueva situación, permitiendo la ejecución de otra respuesta. También puede demorarse temporalmente, esperando un momento posterior más adecuado para su puesta en práctica.

La investigación de las funciones ejecutivas cerebrales ha despertado, en los últimos años, un creciente interés (Sánchez y Narbona, 2001). Son abundantes los trabajos que estudian sus alteraciones en distintas enfermedades, especialmente en el trastorno por déficit de 
atención e hiperactividad (Barkley, 1997; Grodzinsky y Diamond, 1992; Seidman, Biederman, Faraone, Weber y Oullette, 1997; Seidman, Biederman, Weber, Hatch y Faraone, 1998).

Así, en esta línea, los estudios clínicos (sobre lesiones) indican que existe una asociación entre la disfunción del córtex prefrontal dorsolateral producida en el síndrome disejecutivo y dificultades atencionales, carencia de comportamiento estratégico o autodirigido, o la capacidad de demorar o inhibir respuestas. La disfunción del córtex orbitofrontal puede desencadenar euforia, desinhibición, hipomanía esporádica, impulsividad y un comportamiento social inadecuado, mientras que la disfunción del cíngulo anterior puede manifestarse como aislamiento social o apatía o una reducción de la vigilancia (Pantelis y Brewer, 1996). Aunque los ganglios basales abarcan cinco circuitos paralelos distintos fronto-subcorticales, abarcan las regiones óculo-motora, motora, dorsolateral, prefrontal, orbital lateral y cingulada anterior (Cummings, 1993). Estas vías paralelas proporcionan un marco común para comprender las similitudes y diferencias de los cambios comportamentales, concretamente a nivel cognitivo, atencional, afectivo y motor, asociados con varias lesiones anatómicas y varios trastornos neurodegenerativos y neuroevolutivos.

\section{Valoración del TDAH}

\section{Valoración comportamental}

Un aspecto clave en la evaluación del TDAH consiste en inferir el funcionamiento cerebral de estos niños a través de sus comportamientos. Para ello, existen en el mercado multitud de listados que hacen referencia a la falta de atención, el exceso de actividad y la impulsivdad (e.g., Listado de criterios del DSM-IV para el diagnóstico del TDAH, Escala de Conners, EDAH, etc.). Cabe señalar la importancia de que dichas escalas sean cumplimentadas por personas que conocen al niño en los contextos naturales en los que más tiempo pasa: la casa y la escuela.

Un factor decisivo en el diagnostico de la hiperactividad consiste en dilucidar si las manifestaciones de inatención, impulsividad y sobreactividad de un sujeto son meros síntomas aislados o constituyen un trastorno. Cualquier niño puede presentar de forma aislada y 
transitorias dichos comportamientos, pero estos síntomas no tienen porqué constituir un trastorno por sí mismos. Por el contrario, el TDAH es un síndrome que cumple la mayoría de los requisitos de un trastorno clínico, es decir, tiene un curso, una duración, y una respuesta al tratamiento que son específicos.

\section{Evaluación a través de padres y profesores:}

La primera fase en la evaluación consiste en realizar una anamnesis minuciosa, recogiendo información de padres y profesores referente a los aspectos prenatales y perinatales del sujeto, así como su curso evolutivo, historia escolar, etc. A continuación mostramos un esbozo de los datos más pertinentes a indagar:

- Historia clínica y evolutiva: Curso del embarazo y posibles factores de riesgo pre y peri-natales que sugieran la existencia de un posible daño neurológico.

- Historia del problema: Interesa conocer cuándo y cómo ha surgido el trastorno, y qué síntomas ha ido manifestando el niño.

- Recopilación de información respecto al clima familiar o situación familiar que tienen: Trabajo de los padres, horarios, organización de las tareas domésticas ya que todos estos aspectos pueden determinar actitudes negativas hacia el hijo e influir sobre la capacidad para poner en práctica las recomendaciones terapéuticas. De igual forma, es interesante indagar sobre las estrategias parentales para el manejo del comportamiento y las distintas reacciones del niño ante las pautas de disciplina.

Posteriormente se puede pasar a analizar la sintomatología y la problemática del niño de forma sistemática utilizando las siguientes escalas comportamentales, basadas en listados generales o específicos, 
Tabla I. Escalas comportamentales para la valoración del TDAH

1- Listas Generales de Problemas: Escala de Problemas de Conducta para padres (EPC, Navarro, Peiró, Llácer y cols, 1993); Inventario de Problemas en la Escuela (IPE, Miranda, Martorell, Llácer y cols, 1993); e Inventario de Síntomas Infantiles de Stony Brook (Gadow y Sprafkin, 1995).

2- Escalas Específicas de Hiperactividad: Escala de Conners para padres y profesores (1982); Escala Iowa-Conners para el profesor (Loney y Milich, 1982); Cuestionario de Autocontrol para padres y profesores (Kendall y Wilcox, 1979); y Cuestionario de Conductas Problemáticas para Preescolares (Miranda y Santamaría, 1986). Escala de déficit de atención e hiperactividad (E.D.A.H.). (Farré y Narbona, 1997).

3- Escalas para evaluar la Variación Situacional: Cuestionario de Situaciones en la Escuela (Barkley, 1981)

Para una información más detallada sobre las distintas escalas, remitimos al lector al manual de Miranda, Roselló y Soriano (1998).

\section{$\underline{\text { Observación directa del comportamiento }}$}

Además de las escalas comportamentales, también resultan muy útiles las técnicas de observación directa del comportamiento en las diferentes actividades que el niño realiza en el colegio y en el hogar. Para ello, se pueden utilizar registros de intervalo temporal (ej. Segmentos de 15 minutos) registrando la presencia o ausencia de determinados comportamientos; registros de análisis de tareas o registros funcionales (antecedentes y consecuentes a una conducta determinada del niño).

Por último, existen una serie de conductas con un alto índice de ocurrencia que deberían registrarse de forma sistemática, especialmente en el contexto escolar. Dichas conductas podrían ser: Estar fuera de la tarea (jugando con el material, realizando otras tareas, no escuchando); no obedecer al profesor; intranquilidad en el asiento (cambiando constantemente de posición, escurrirse en el asiento); molestar al profesor o a los compañeros (gritar, interrumpir a los demás cuando hablan, hacer ruidos inapropiados, hacer el payaso); levantarse del sitio; o agresividad (dar patadas, morder, pegar, poner la zancadilla, dañar la propiedad de otras personas. insultar hacer gestos amenazantes, amenazas verbales, contestar de forma inaceptable) 
En resumen, en el proceso de evaluación del TDAH será conveniente incluir múltiples medidas que recojan la variedad de síntomas presentes en el trastorno y aporten información útil sobre la variedad de problemas que suelen aparecer asociados. Se trata en cualquier caso de adoptar una perspectiva funcional, centrándose en los déficits o excesos del funcionamiento y analizando la naturaleza de estos problemas de cara a seleccionar las técnicas de intervención más adecuadas de acuerdo con la información que se obtenga.

\section{Valoración del funcionamiento ejecutivo}

Muchos autores han establecido un sorprendente paralelismo entre los comportamientos de los niños con TDAH y los comportamientos de los adultos con daño cerebral frontal (Smith, Kates y Vriezen, 1992; Shue \& Douglas, 1992). Las dificultades de los niños con TDAH en edad escolar parecen centrarse en el dominio de comportamientos asociados con el funcionamiento frontal; es decir, la auto-regulación o funciones ejecutivas tales como fluidez verbal, impulsividad, pérdida de la inhibición y el mantenimiento de la atención, así como la organización y el monitoreo de la conducta (Barkley, 1997; Pennington \& Ozonoff, 1996, Shue \& Douglas, 1992).

La evaluación del funcionamiento ejecutivo (FE) constituye uno de los grandes retos en la investigación actual del TDAH. Una de las mayores dificultades con las que se ha encontrado este campo ha sido la forma de diseñar y aplicar las distintas tareas experimentales, así como la validez discriminante de las tareas. Por otra parte, existen estudios que hablan de la baja correlación entre informes y auto-informes con medidas de laboratorio (ver White, Moffit, Caspi y cols., 1994). Otro aspecto crítico resulta de que la mayoría de investigación sobre FE procede de estudios sobre adultos con lesiones frontales, por lo que es necesario la adaptación de las distintas tareas utilizadas en estos grupos experimentales a niños.

Partiendo de todas las dificultades descritas anteriormente, remitimos al lector al trabajo experimental realizado por Félix (2003). En él, se analizan sucintamente un conjunto de tareas neuropsicológicas, que tras un estudio experimental en varios colegios, utilizando muestras de niños con edades comprendidas entre los 6 y los 12 años, realiza una selección de aquellas tareas que son susceptibles de utilizarse con niños. Posteriormente, realizó un estudio comparativo en la ejecución de dicha muestra escolar con niños diagnosticados de TDAH, obteniendo un potente factor que aglutinó una serie de tareas computerizadas relacionadas 
directamente con el control inhibitorio, correlacionando máximamente con las estimaciones comportamentales de padres y profesores. La ejecución de las tareas discriminó de forma significativa a aquellos niños con TDAH, constituyendo así una batería neuropsicológica específica para la evaluación de la hiperactividad. Dichas tareas (o adaptación de las mismas), las está utilizando este autor en un grupo de investigación en el Hospital Clínico de Valencia, junto con técnicas de Resonancia Magnética Funcional y estudios genéticos en niños con TDAH.

Existen en la actualidad multitud de tareas que de una forma u otra, están dirigidas a la valoración de alguno de los procesos controlados por el funcionamiento ejecutivo. En la Tabla II mostramos algunas de ellas.

Tabla 2.-

\begin{tabular}{|c|c|c|}
\hline FUNC. EJECUTIVO & TEST & VERSIÓN \\
\hline $\begin{array}{l}\text { FLEXIBILIDAD } \\
\text { COGNITIVA }\end{array}$ & $\begin{array}{l}\text { Wisconsin Card Sorting } \\
\text { Test (WCST) }\end{array}$ & $\begin{array}{l}\text { (Grant \& Berg, 1984; Heaton, } 1981 \\
\text { Tarea de ordenador (ver Félix, 2003) }\end{array}$ \\
\hline PLANIFICACIÓN & Torre de Londres (TOL) & $\begin{array}{l}12 \text { ensayos + } 1 \text { entrenamiento: } 2 \text { x ( } 2 \\
\text { y } 3 \text { movimientos), } 4 \text { x (4 y } 5 \text { movi- } \\
\text { mientos) } \\
\text { (Shallice, 1982) }\end{array}$ \\
\hline $\begin{array}{l}\text { MEMORIA DE TRA- } \\
\text { BAJO }\end{array}$ & $\begin{array}{l}\text { Working Memory- } \\
\text { Sentences } \\
\\
\text { Temporo-Spatial Recall } \\
\text { Task }\end{array}$ & $\begin{array}{l}15 \text { ensayos: } 3 \text { x }(2,3,4,5 \text { o } 6 \text { frases) } \\
\text { (Siegel \& Ryan, 1989) } \\
\text { Total } 30 \text { ensayos }+3 \text { entrenamiento: } \\
6 \text { x }(2,3,4,5 \text { o } 6 \text { ítems) (Dubois \& } \\
\text { cols., } 1995) \\
\text { Tarea de ordenador }\end{array}$ \\
\hline $\begin{array}{l}\text { CONTROL INHIBI- } \\
\text { TORIO }\end{array}$ & $\begin{array}{l}\text { Stroop Test } \\
\text { Stop Task } \\
\\
\text { Test de Emparejamiento } \\
\text { de Figuras Familiares } \\
\text { (MFFT) }\end{array}$ & $\begin{array}{l}\text { Palabra, Color y Color-Palabra } \\
\text { (Stroop, 1935) } \\
\text { (Logan \& Cowan, 1984 Adaptación } \\
\text { de Avila \& Parcet, 2001) } \\
\text { Tarea de ordenador (ver Félix, } \\
\text { 2003) } \\
\text { Versión de Servera y Llabrés (2000) } \\
\text { Tarea de ordenador (ver Félix, } \\
2002 \text { y 2003) }\end{array}$ \\
\hline AUTO-MONITOREO & Fluidez verbal & $\begin{array}{l}\text { Categorías fonológicas y semánticas: } \\
\text { Animales \& frutas, palabras que em- }\end{array}$ \\
\hline
\end{tabular}




\begin{tabular}{||l|l|l||}
\hline \hline & $\begin{array}{l}\text { piezan por P \& S (Benton \& Hams- } \\
\text { her, 1978) }\end{array}$ \\
\hline ATENCIÓN & $\begin{array}{l}\text { Continuous Performan- } \\
\text { ce Test (CPT) }\end{array}$ & $\begin{array}{l}\text { (Adaptación de Avila \& Parcet, } \\
\text { 2001) } \\
\text { Tarea de ordenador (Félix, 2003) }\end{array}$ \\
\hline
\end{tabular}

Pese a la proliferación de tareas neuropsicológicas, muchas veces nos encontramos ante la dificultad de no disponer de tiempo suficiente para el pase de todas ellas. Por ello, existe un trabajo realizado desde el contexto hospitalario (Andrés, Castelló, Félix y Castillo, 2005) donde se plantea el uso de sólo dos tareas neuropsicológicas (Tarea de Stop y CPT) como buenos indicadores para el diagnóstico del TDAH. Estos autores realizaron un estudio con 30 niños, donde utilizaron estas dos tareas junto con estimaciones comportamentales, obteniendo una correlación significativa entre los niños evaluados y la respuesta al tratamiento farmacológico con metilfenidato.

\section{Nuevas tendencias: Resonancia Magnética Funcional como ventana al cerebro}

La RMf como una ventana al cerebro "en acción”, puede proporcionar interesantes aportaciones sobre "cómo" se ejecutan las tareas cognitivas. Es decir, la RMf puede informar sobre estrategias y mecanismos compensatorios que no son observables con el sólo uso de herramientas neuropsicológicas.

Como ventanas al cerebro, las técnicas de neuroimagen funcional indudablemente pueden destacar los mecanismos que no son visibles desde la psicología. A partir del estudio de los patrones de activación que corresponden a la ejecución de una tarea particular, se proporciona información más profunda sobre las diferencias en la estrategia (por ejemplo, a través de la incorporación de diferentes regiones cerebrales) o sobre los patrones de activación compensatorios, empleados por un grupo de pacientes para enfrentarse a una tarea que es particularmente difícil para ellos. Determinar los déficits neuropsicológicos específicos a nivel de ejecución y los patrones de activación correspondientes a dicho trastorno, puede brindar luces importantes para comprender la neuro-cognición de un trastorno particular y, además, proporcionar mayores aportes sobre las estrategias de ejecución y los mecanismos compensatorios a nivel psicológico, así como del uso de regiones anatómicas definidas a nivel cerebral. 
Un estudio reciente de Schweitzer y sus colaboradores (Schweitzer, Fabel, Grafton et al., 2000) demostraron la incorporación de vías neuronales inusuales en adultos con TDAH mientras organizaban estrategias menos eficientes para resolver una tarea de memoria de trabajo. Una vez más, los datos de imaginería cerebral apuntan hacia las diferencias en las estrategias de pacientes y controles; que se sospechaban, pero no habían sido evidenciadas directamente con la neuropsicología.

La RMf también ha sido de utilidad para dar validez a hipótesis planteadas a partir de la neuropsicología. En el caso del TDAH, los estudios de RMf han confirmado predicciones que parten de observaciones neuropsicológicas. Así, a partir de la imaginería funcional se ha confirmado la hipótesis de los déficits en la incorporación de estructuras frontales durante la ejecución de tareas de funcionamiento ejecutivo de los lóbulos frontales (Rubia,Taylor, Smith et al., 2001; Vaijdya, Austin, Kirkonian et al., 1998). En la misma muestra de adolescentes hiperactivos, se observaba una activación frontal reducida durante la ejecución de tareas de alto nivel de funcionamiento ejecutivo, pero no en una tarea de "finger tapping" de bajo nivel; con lo cual, parece confirmarse la especificidad de los déficits ejecutivos (Rubia, et al., 2001). Asimismo, la hipótesis de las anormalidades en la incorporación de la región fronto-estriada pudo ser confirmada durante la ejecución del stop task (Rubia et al.2000, 2001).

Estudios futuros de RMf que comparen el TDAH con otros grupos de pacientes serán importantes para centrarse en la especificidad de los hallazgos de la hipofrontalidad y su asociación con la especificidad de los déficit en la impulsividad. Han aparecido recientemente en la literatura científica los trabajos de la investigadora Katya Rubia que apuntan en esta dirección. Por ejemplo, en un reciente trabajo (Rubia, 2002), comparó la ejecución en la tarea de stop dos grupos psiquiátricos: por un lado, pacientes diagnosticados de TDAH y por otro, pacientes esquizofrénicos. Ambos grupos mostraron diferencias significativas en los patrones de activación durante la ejecución de la Tarea de Stop. Así, los sujetos con TDAH mostraron una baja activación en el hemisferio derecho, concretamente en el cingulado anterior, y en el córtex prefrontal inferior y dorsolateral, el grupo de esquizofrénicos mostró una activación reducida del hemisferio izquierdo en las mismas zonas, aunque con sobre-actividad en el hemisferio derecho, concretamente en el tálamo y en el caudado. Por lo tanto, la lateralidad de la baja activación de la zona prefrontal fue inversa, al igual que se produjo una sobreactivación de las regiones del caudado en esquizofrénicos y una baja activación de esta zona en los sujetos con TDAH. La sobre-activación en las áreas subcorticales en la esquizofrenia podría reflejar un mecanismo de inhibición alternativo que no está presente en los sujetos con 
TDAH, y que podría explicar la no existencia de déficits en la ejecución de la tarea por parte de los sujetos esquizofrénicos.

Estos hallazgos derivados del trabajo de Rubia (2002) son interesantes de cara a la verificación y réplica de hipótesis planteadas con anterioridad de cara a ambos trastornos. Así, en el caso del TDAH, se confirma a través de las técnicas de neuroimagen los déficits en la activación y estructura que se habían centrado en la zona derecha de los lóbulos frontales (Rubia y Smith, 2001; Santosh, 2000; Swanson et al., 1998), mientras que los déficits funcionales y estructurales de la esquizofrenia han sido localizados de forma más predominante en la zona izquierda frontal y áreas temporales (Harris, 1999).

\section{Conclusiones}

Tras el análisis de las distintas formas de abordar la evaluación del TDAH, queda claro que es necesario recopilar información de distintas vías, principalmente de la historia del niño, de los aspectos comportamentales en contextos naturales (casa y escuela) y del desempeño neuropsicológico de los distintos dominios del funcionamiento ejecutivo.

Por lo que respecta a la utilización de una u otra forma de evaluación, pensamos que el diagnóstico de TDAH no puede realizarse a partir de las puntuaciones obtenidas en una escala comportamental, siendo necesario indagar sobre los otros aspectos anteriormente comentados. De igual forma, aunque la validez de las tareas neuropsicológicas es aceptable (ver Miranda, Félix y Ávila, en prensa), todavía queda un largo camino por recorrer. La RMf constituye una buena herramienta para la investigación, permitiendo detectar procesos de activación de las distintas áreas del cerebro en vivo, mientras el sujeto realiza las distintas tareas neuropsicológicas, aunque todavía requiere de más investigación para utilizarse como una prueba diagnóstica.

En resumen, son distintos los dominios que dentro del TDAH pueden aparecer alterados (cognitivo, comportamental, social, etc.). De igual forma, los trastornos comórbidos juegan un papel esencial en el proceso de evaluación, ya que su delimitación permitirá una intervención (tanto psicopedagógica como farmacológica) más ajustada a las necesidades de estas personas. Por lo tanto, queda todavía un largo camino por recorrer en el diseño de tareas es- 
pecíficas para la valoración del TDAH, necesitando de un buen corpus teórico y de evidencia empírica para su uso generalizado.

\section{Referencias}

American Psychiatric Association. (2002). Manual Diagnóstico y Estadístico de los Trastornos Mentales. DSM-IV-TR. Barcelona: Masson.

Andrés, M., Castelló, M.L., Félix, V. y Castillo, S. (2005). Valoración del TDAH en una consulta de neuropediatría: Aspectos clínicos y neuropsicológicos. Comunicación presentada en la Sociedad Valenciana de Pediatría. Castellón, junio de 2005.

Avila, C. y Parcet, M.A. (2001). Personality and inhibitory deficits in the stop-signal task: The mediating role of Gray's anxiety and impulsivity. Personality and Individual Differences, 29, 875-986.

Barkley, R.A. (1981). Hyperactivity. En E. Mash y L. Terdal (Eds.), Behavioral Assessment of Childhood Disorders. New York. Guilford Press.

Barkley, R.A. (1997). Behavioral inhibition, sustained attention and executive functions: Constructing a unifying theory of ADHD. Psychological Bulletin, 121, 65-94

Benton, A.L. y Hamsher, K. (1978). Multilingual aphasia examination. Iowa City: University of Iowa.

Clements, S.D. (1966). Minimal brain dysfunction in children: terminology and classification. NINDB Monograph, $n^{\circ}$ 3. Washington D.C.: Public Health Service.

Conners, C.K. (1982). Parent and teacher rating forms for the assessment of hyperkinesis in children. En P.A Keller y L.J. Ritt (Eds.), Innovations in clinical practice: A source book (Vol. I). Sarasota FL: Professional Resource Exchange.

Cummings, J.L. (1993). Frontal-subcortical circuits and human behaviour. Archives of Neurology, 50, 873-80.

Douglas, V.I. (1972). Stop, look and listen: the problem of sustained attention and impulse control in hyperactive and normal children. Canadian Journal of Behavioral Science, 4, 259-282.

Dubois B, Levy R, Verin M, Teixeira-Ferreira C, Agid Y, Pillon B Experimental approach to prefrontal functions in humans. Annals of the New York Academy of Sciences, 1995, $769: 41-60$ 
Farré, A. y Narbona, J (1997). Escala de déficit de atención e hiperactividad (E.D.A.H.). Madrid. TEA.

Félix, V. (2002). Evaluación del control inhibitorio a través de la Tarea de Stop. Tesina. Universidad de Valencia.

Félix, V. (2003). Evaluación de la impulsividad en el trastorno por déficit de atención con hiperactividad a través de tareas neuropsicológicas. Tesis Doctoral. Universidad de Valencia.

Gadow, K. y Sprafkn, J. (1995). Child Sympton Inventories. The revised CSI Parent/Teacher Checklist Checkmate Plus, LTD. Stony Brook, NY.

Gazzaniga, M.S., Ivry, R.B. y Mangun, G.R. (2001). Cognitive Neuroscience. The biology of mind. $2^{\text {nd }}$ Ed. W.W. Norton \& Company. New York, London.

Grant, D.A. y Berg, E.A.A. (1984). A behavioral analysis of degree of reinforcement and ease of shifting to new responses in a Weigel-type card-sorting problem. Journal of Experimental Psychology, 38, 404-411.

Grodzinsky, G.M, y Diamond, R., (1992). Frontal lobe functioning in boys with attention-deficit hyperactivity disorder. Developmental Neuropsychology, 8, 427-446.

Harris, L.J. (1999). Early theory and research on hemispheric specialization. Schizophrenia Bulletin, 25, 11-39.

Heaton, R.K. (1981). Wisconsin Card Sorting Test Manuel. Odessa (FL). Psychological Assessment Resource Inc.

Kendall, P. y Wilcox, L.E. (1979). Self-control in children: Development of a rating scale. Journal of Consulting and Clinical psychology, 47, 1020-1029.

Logan, G.D. y Cowan, W.B. (1984). On the ability to inhibit thought and action: A theory of an act of control. Psychological Review, 91, 295-327.

Loney, J., Milich, R. (1982). Hiperactivity, Inatention and aggression in clinical practise. En: M. Wolraich y D.K. Routh (Eds.), Advances in behavioral pediatrics, $2^{\text {nd }}$ ed. Greenwich: JAI Press. (pp. 113-147).

Luria, A.R. (1973). The working Brain. London: Penguin Books.

Miranda, A. y Santamaría, M. (1986). Hiperactividad y Dificultades en el Aprendizaje. Valencia. Promolibro.

Miranda, A., Félix, V. y Ávila, C. (en prensa). Validez de las tareas neuropsicológicas. Psicología General y Aplicada. 
Miranda, A., Martorell, C., Llácer, M. D., Peiró, R. y Silva, F. (1993). IPE. Inventario de Problemas en la Escuela. En F. Silva y C. Martorell (Comp.). Evaluación de la Personalidad Infantil y Juvenil. EPIJ(Vol 1). Madrid.MEPSA.

Miranda, A., Roselló, B. y Soriano, M. (1998). Estudiantes con deficiencias atencionales. Valencia: Promolibro.

Navarro, A., Peiró, R., Llácer, M.D. y Silva, F. (1993). Escala de problemas de conducta (EPC), en F. Silva y M.C. Martorell (eds.), EPIJ. Evaluación de la Personalidad Infantil y Juvenil. Madrid. TEA

Ozonoff, S., Strayer, D.L., McMahon, W. y Filloux, F. (1994). Executive function abilities in autism and Tourette syndrome: an information processing approach. Journal of Child Psychology and Psychiatry, 35, 1015-1032.

Pantelis, C. y Brewer, W. (1996). Neurocognitive and neurobehavioral patterns and the syndromes of schizophrenia: Role of frontal-subcortical networks. En C. Pantelis, H.E. Nelson y T.R.E. Barnes (Eds.), Schizophrenia: A neuropsychological perspective (pp. 317-343). Chichester: Wiley.

Pennington, B.F. y Ozonoff, S. (1996). Executive functions and developmental psychopathology. Journal of Child Psychology and Psychiatry, 37, 51-87.

Rubia, K. (2002). The dynamic approach to neurodevelopmental psychiatric disorders: use of fMRI combined with neuropsychology to elucidate the dynamics of psychiatric disorders, exemplified in ADHD and schizophrenia. Behavioural Brain Research, 130, 4756.

Rubia, K., Overmeyer, S.O., Taylor, E., Brammer, M., Williams, S., Simmons, A., Andrew, C. y Bullmore, E.T. (2000). Frontalisation with age: mapping neurodevelopmental trajectories with fMRI. Neurosciencie Biobehavior Review, 24, 13-19.

Rubia, K., Taylor, E., Smith, A., Oksanen, H., Overmeyer, S., Bullmore, E.T. y Newman, S. (2001). Neuropsychological analyses of impulsiveness in childhood hyperactivity. British Journal of Psychiatry, , 179, 138-143.

Sánchez, R. y Narbona, J. (2001). Revisión conceptual del sistema ejecutivo y su estudio en el niño con trastorno por déficit de atención e hiperactividad. Revista de Neurología, 33, 47-53.

Santosh, P.J. (2000). Neuroimaging in child and adolescent psychiatric disorders. Arc.Dis. Child., 82,(5), 412-419. 
Schweitzer, J.B., Fabel, T.L., Grafton, S.T., Tune, L.E., Hoffman, J.M. y Kilts, C.D. (2000). Alterations in the functional anatomy of working memory in adult attention deficit hyperactivity disorder. American Journal of Psychiatry, 157, 278-280.

Seidman, L.L., Biederman, J., Faraone, S.V., Weber, W. y Oullette, C. (1997). Towards defining a neuropsychology of Attention Deficit-Hyperactivity Disorder: Performance of children and adolescents from a large clinically referred sample. Journal Consulting of Clinical Psychology, 65, 150-160.

Seidman, L.R., Biederman, J., Weber, W., Hatch, M. y Faraone, S.V. (1998). Neuropsychological function in adults with attention-deficit hyperactivity disorder. Biological Psychiatry, 44, 260-8.

Servera, M. \& Llabrés, J. (2000). EMIC (Escala Magallanes de Impulsividad Computarizada). Bilbao: Albor-Cohs.

Shallice, T. (1982). Specific impairments of planning. Philosophical Transactions of the Royal Society of London, B, 298, 199-209.

Shue, K.L. y Douglas, V.I. (1992). Attention deficit hyperactivity disorder and the frontal lobe syndrome. Brain and Cognition, 20, 104-124.

Siegel, L.S. y Ryan, E.B. (1989). The development of working memory in normally achieving and subtypes of learning disabled children. Child Development, 60, 973-980.

Smith, M.L., Kates, M.H. y Vriezen, E.R. (1992). The development of frontal-lobe functions. In S.J. Segalowitz y I. Rapin (Eds.), Handbook of Neuropsychology, Vol. 7: Child Nueropsychology (pp. 309-330). Amsterdam: Elsevier Science Publishers B.V.

Still, G.F. (1902). Some abnormal physical conditions in children. Lancet, 1, 1008-1012.

Stroop, J.R. (1935). Studies of interference in serial verbal reactions. Journal of Experimental Psychology, 18, 643-662.

Stuss, D.T. (1992). Biological and physiological development of executive functions. Brain and Cognition, 20, 8-23.

Vaijdya, C.J., Austin, G., Kirkorian, G., Ridlehuber, H.W., Desmond, J.E., Glover, G.H. y Ganrieli, J.D.E. (1998). Selective effects of methylphenidate in attention deficit hyperactivity disorder: a functional magnetic resonance study. Proceedings of the $\mathrm{Na}$ tional Academy of Science USA, 95, 14994-14999.

White, J.L., Moffitt, T.E., Caspi, A., Bartusch, D.J., Needles, D.J., y Stouthamer-Loeber, M. (1994). Measuring impulsivity examining its relationship to delinquency. Journal of Abnormal Psychology, 103, 192-205. 
Perspectivas recientes en la evaluación neuropsicológica y comportamental del Trastorno por Déficit de Atención con Hipeactividad

[Pagina en blanco por razones de paginación] 